\title{
Flicker and suprathreshold spatial summation: Evidence for a two-channel model of achromatic brightness
}

\author{
BRUCE DRUM \\ George Washington University, Washington, D.C.
}

\begin{abstract}
Spatial summation of brightness was measured in the light-adapted periphery for targets that either flickered at $20 \mathrm{~Hz}$ or remained steady during a 1-sec exposure. For each target condition, stimuli of different diameters were adjusted in intensity to match a constant foveal reference target in subjective brightness. For comparison, increment thresholds were also measured. Large differences were found among the resulting equal-brightness functions of three normal observers. The data are consistent with a two-channel model of achromatic brightness. The spatial and temporal properties of the two brightness channels resemble those of the tonic and phasic systems of electrophysiology.
\end{abstract}

The characteristics of threshold spatial summation for circular targets are well known. The usual result is that threshold decreases in proportion to stimulus area out to some "critical" size, beyond which threshold decreases at a progressively lower rate and eventually becomes independent of area.

Spatial summation can also be measured above threshold by matching the brightness of a variable-size test target to that of a simultaneously presented reference target of constant size and luminance. The luminances of the brightness matches can then be plotted as a function of stimulus area to form "equal-brightness" curves. These brightness spatial summation functions differ from the analogous threshold curves in that they often do not decrease monotonically with increasing size, but turn upward again before finally leveling off (Higgins \& Rinalducci, 1975). This reversal strongly resembles the Broca-Sulzer effect (Broca \& Sulzer, 1902), in which a similar brightness reversal occurs as a function of stimulus duration. Higgins and Rinalducci (1975) have accordingly named the spatial brightness reversal the "spatial Broca-Sulzer effect."

Drum (1980) studied the spatial summation of brightness in the peripheral visual field under a variety of adaptation conditions. The relative shapes and positions of threshold and suprathreshold spatial summation functions changed systematically with adaptation. Differences be-

Supported by NIH Grants EY01672 and EY03710. I am grateful to William Huppert and Fareed Armaly for technical assistance, and to Frederick Kitterle for very helpful comments on an early draft of the manuscript. A preliminary report of part of this research was presented at the 1978 annual meeting of the Optical Society of America, in San Francisco. The author's current mailing address is: Laboratory of Physiological Optics, Wilmer Ophthalmological Institute, B-27, Johns Hopkins University School, 601 North Broadway, Baltimore, MD 21205. tween observers were small for the threshold functions but large for the equal-brightness functions. The brightness results ranged from pronounced spatial Broca-Sulzer effects, with reversal diameters that increased with dark adaptation, to monotonic functions that ran nearly parallel to the threshold functions for all adaptation conditions.

These results, among others, suggest a two-channel model for achromatic brightness (see Drum, 1980, for a full discussion). Briefly, the model postulates "strong" and "weak" brightness channels for which equal neural responses produce unequal brightness sensations; a justdetectable signal mediated by the strong channel appears much brighter than a just-detectable signal mediated by the weak channel. The two channels also have different spatial properties, with the strong channel maximally sensitive to small stimuli and the weak channel maximally sensitive to large stimuli. Furthermore, the weak channel is paradoxically more sensitive than the strong channel, except sometimes for very small stimuli. For each channel, however, the brightness signal corresponding to a given response level is assumed to be independent of stimulus size.

The above model can explain the previous brightness spatial summation data by means of shifts in the relative sensitivities of the strong and weak channels. If the weak channel is sensitive enough to exclusively determine both threshold and brightness near threshold at all target sizes, then brightness spatial summation curves that are near enough to threshold should run parallel to the threshold spatial summation curve. If small targets are seen partially by the strong channel, however, their brightness is selectively increased, giving rise to a spatial Broca-Sulzer effect.

Drum (1980) pointed out several apparent similarities between the strong and weak brightness channels and the tonic and phasic neural systems reported in numerous elec- 
trophysiological studies ${ }^{1}$ (for a recent review, see Lennie, 1980). Both tonic and phasic neurons have center-surround receptive field organization. However, tonic cells are relatively sensitive to small stimuli and insensitive to large stimuli, like the strong channel, whereas phasic cells are relatively sensitive to large stimuli, like the weak channel (Cleland, Levick, \& Sanderson, 1973). Also, phasic cells appear to be more sensitive than tonic cells to spatially optimal stimuli (Hammon \& Scobey, 1982), suggesting that phasic cells may determine increment thresholds in most situations.

Tonic and phasic neurons differ in their temporal as well as their spatial properties. Tonic cells tend to respond in a sustained fashion to steady stimuli and are most sensitive to low temporal frequencies, whereas phasic cells tend to respond transiently at stimulus onset and are most sensitive to intermediate temporal frequencies. Also, tonic cells respond to flicker by modulating their discharge rate about a constant mean level, whereas the phasic cell flicker response may include large increases of the average discharge rate (DeMonasterio, 1978; Fukada \& Saito, 1971; Victor \& Shapley, 1979).

If the proposed strong and weak brightness channels are mediated by tonic and phasic cells, their temporal responses should differ like those of tonic and phasic cells. For example, the strong channel should be relatively insensitive to medium- or high-frequency flicker, and flicker should selectively increase the weak-channel brightness signal.

To test the above predictions, the present study compared spatial summation of brightness for flickering and nonflickering targets. A $20-\mathrm{Hz}$ frequency was chosen for the flickering targets in order to preferentially stimulate the phasic system. Brightness spatial summation functions measured with flickering stimuli were therefore expected to reflect more weak-channel input than functions measured with nonflickering stimuli. This expectation was confirmed.

\section{METHOD}

\begin{abstract}
Apparatus and Stimuli
The apparatus was a Tübingen perimeter (Sloan, 1971), modified to provide increased background and test stimulus luminance. All stimuli were projected into a white hemispherical bowl with a radius of $33 \mathrm{~cm}$. The stimuli were viewed monocularly from the center of the hemisphere. The global background had a luminance of $38 \mathrm{~cd} / \mathrm{m}^{2}$ at an approximate color temperature of $2800^{\circ}$. The test stimuli had a color temperature of $3400^{\circ}$, and they ranged in diameter from $7^{\prime}$ to $104^{\prime}$ of visual angle. The test stimulus was either a $1-\mathrm{sec}$ steady flash or a $1-\mathrm{sec}$ burst of $20-\mathrm{Hz}$ square-wave flicker. The test position was at $20^{\circ}$ eccentricity in the nasal visual field. The fixation target was a small white spot $\left(10^{\prime}\right.$ diameter, $3000^{\circ}$ color temperature) that also served as a fixed luminance brightness reference in the brightness-matching part of the experiment.
\end{abstract}

\section{Subjects}

Three young males (aged 20,31 , and 34 ) with normal visual acuity, visual fields, and color vision served as subjects. One subject was emmetropic and two were moderately myopic; all three could easily accommodate to the $33-\mathrm{cm}$ viewing distance without refractive corrections. Pupil size was not controlled, but was measured in situ at the beginning of each session. Pupil diameter was approximately $4 \mathrm{~mm}$ for all subjects. The subjects were all highly practiced psychophysical observers and were aware beforehand of the hypothesis being tested.

Previous tests of the "steady" condition had found large individual differences in spatial brightness summation. The present subjects were selected to span, as nearly as possible, the entire range of these differences: Subject B.D. consistently had shown a pronounced spatial BrocaSulzer effect, W.H. had shown a relatively weak one, and F.A. had shown only a slight divergence of his threshold and brightness functions with no brightness reversal at all.

\section{Procedures}

Increment thresholds and brightness matches to the foveal reference were measured as a function of stimulus size for both flickering and steady targets. Measurements were always completed in order of increasing stimulus size, and all four measurements were made at each size before proceeding to the next one. For each size, the measurement order was: (1) steady increment threshold, (2) steady brightness match, (3) flickering increment threshold, and (4) flickering brightness match.

The threshold of the foveal reference target was measured at the start of each session. The reference luminance for the brightness matches was then set by temporarily fixing the luminance of the smallest steady test stimulus at $0.3 \mathrm{log}$ unit above its threshold and adjusting the reference luminance in a "reverse matching" procedure. This ensured that all of the steady brightness-matching functions would be close to $0.3 \log$ unit above threshold at the smallest stimulus size.

All procedures were identical for flickering and steady targets. Although thresholds for flicker detection were not formally measured, the subjects were frequently asked to report on the subjective appearance of the test target, including whether or not flicker was visible in the flicker conditions.

Thresholds were measured by a method of descending limits. Starting at a clearly detectable stimulus luminance, the experimenter decreased the luminance by $0.1 \log$ unit after each "yes" response and kept it the same after each "no" response. The first luminance to elicit three successive "no" responses was taken as threshold. The median of three such measurements was the overall threshold estimate for the session.

Brightness judgments were based as much as possible on the "steadystate" appearance of the test stimulus; that is, the subjects were instructed to ignore any onset or offset transients.

Brightness matches were measured by the following constant stimulus method. First, the experimenter found a $0.3-0.5-\log$-unit luminance range that included the match. Then he presented a pseudorandom stimulus sequence containing five stimuli at each of several luminance levels within this range (4-6 levels at $0.1-\log$-unit intervals). After each presentation, the subject indicated whether the test stimulus appeared brighter than, equal to, or dimmer than the reference. ${ }^{2}$ These responses were assigned values of 1,0 , and -1 , respectively, and the values for each luminance level were summed. The luminance corresponding to a sum of zero (found by linear interpolation if necessary) was then taken to be the brightness match.

\section{RESULTS AND DISCUSSION}

Figures 1-3 show the average results of three sessions for each of the three subjects. The steady-condition brightness-matching data (upright triangles) confirm that B.D. has a large spatial Broca-Sulzer effect, W.H. has a small one, and F.A. has virtually none. Also in agreement with earlier data (Drum, 1980; Drum, unpublished observations), it was necessary to set the reference luminance much farther above threshold for B.D. than for W.H. or F.A. This is consistent with the subjective reports of the subjects. All three subjects reported that the reference target appeared very dim near threshold. However, although F.A. and W.H. found all of the threshold test targets to be about as bright as the threshold reference target, B.D. 


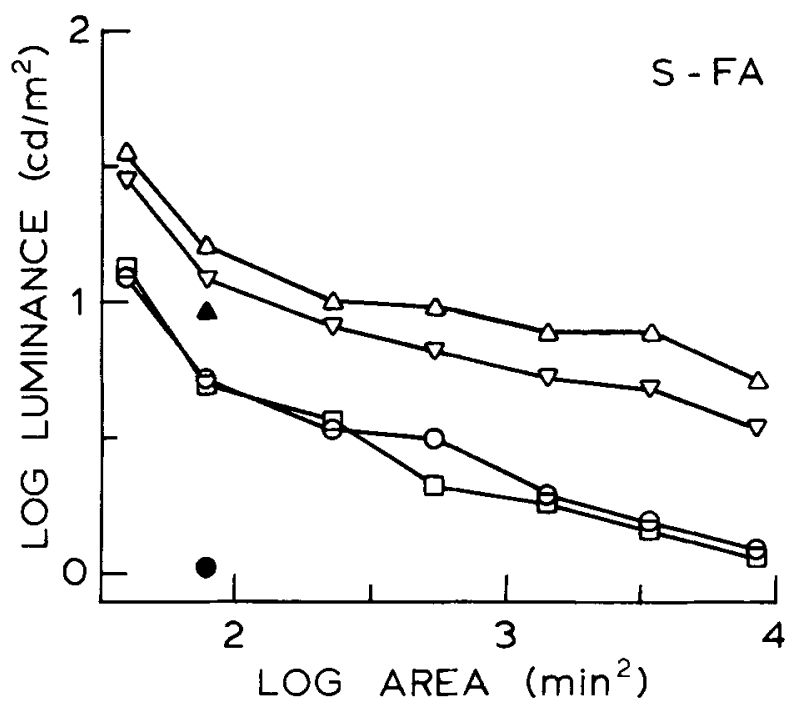

Figure 1. Averaged data from three sessions for Subject F.A. Log increment thresholds and $\log$ brightness matches are plotted as functions of $\log$ stimulus area. Increment thresholds are indicated by circles (1-sec flash) and squares (1-sec burst of 20-Hz flicker), and brightness matches are indicated by upright triangles (1-sec flash) and inverted triangles (1-sec burst of 20-Hz flicker). The log of timeaverage luminance is plotted for all fickering stimuli. The filled circles and upright triangles indicate the thresholds and matching luminances, respectively, for the foveal reference stimulus.

found the smallest test targets to be much brighter than the reference. Assuming that the subjects all perceived the threshold reference target to be about equally bright, these observations imply that small, threshold test targets appeared much brighter to B.D than to W.H. or F.A.

Flicker affects both the threshold and suprathreshold functions in ways that differ for the three subjects. In Figure 1, the flickering brightness function shows a slight, but consistent, drop below the steady brightness function, implying that flicker made stimuli of all sizes appear slightly brighter to F.A. This is the predicted result, given that brightness is determined mainly by the weak (phasic) channel for all target sizes.

It is not clear whether or not flicker had any effect on F.A.'s threshold function. Thresholds for the flickering target average $0.03 \log$ unit below the comparable steady target thresholds (the same direction as the brightness function shift), but the difference is not quite statistically significant [one-tailed paired-comparison $t$ test, $t(20)=$ $1.32, p=.10]$. If only the four largest target sizes are considered, the average difference of $0.067 \mathrm{log}$ unit is marginally significant $[\mathrm{t}(11)=2.15, \mathrm{p}=.03]$, but a single target size is responsible for most of the difference.

Unlike Figure 1, the flickering brightness curve in Figure 3 is essentially equal to the steady brightness curve for small targets, but it drops by as much as $0.5 \mathrm{log}$ unit below the steady curve for large targets. Flicker thus had no effect on B.D.'s brightness perception for small targets, but it made large targets appear considerably brighter. This is the predicted result if the brightness of small stimuli is determined by the strong (tonic) channel. At small sizes, the flicker presumably has no effect because the strong channel cannot resolve it. At large sizes, the strong channel is so insensitive that the weak channel dominates the overall brightness signal. To appear as bright as the strong channel response did at small sizes, however, the weak channel response must be driven much farther above its threshold. At such high response levels, the flicker produces a substantial brightness increase in the weak channel.

Flicker has no appreciable effect on threshold in Figure 3. The two threshold functions do not appear to differ systematically in shape, and their averages are equal to within $0.01 \log$ unit.

The brightness results in Figure 2 appear to be intermediate between those in Figures 1 and 3 . Flicker lowered the entire brightness curve, as in Figure 1, but did so mostly at large stimulus sizes, as in Figure 3 . This result might be expected when both the strong and weak channels contribute significantly to brightness at small sizes. Flicker can then produce a measurable weak-channel brightness increase at small sizes, but not as much as at large sizes where the strong channel influence is greatly reduced.

Surprisingly, W.H.'s threshold function was affected more by flicker than was that of either of the other subjects. Flicker lowered thresholds by an average of $0.06 \mathrm{log}$ unit, which is significant at the .01 level [one-tailed pairedcomparison $\mathrm{t}$ test, $\mathrm{t}(20)=2.55, \mathrm{p}=.01]$. Although there is some suggestion that the greatest threshold drop occurs at the largest size, the main effect appears to be a uniform downward shift of the entire function. This result, as well as the difference between W.H.'s brightness functions at small target sizes, is discussed further below in relation to the question of flicker visibility.

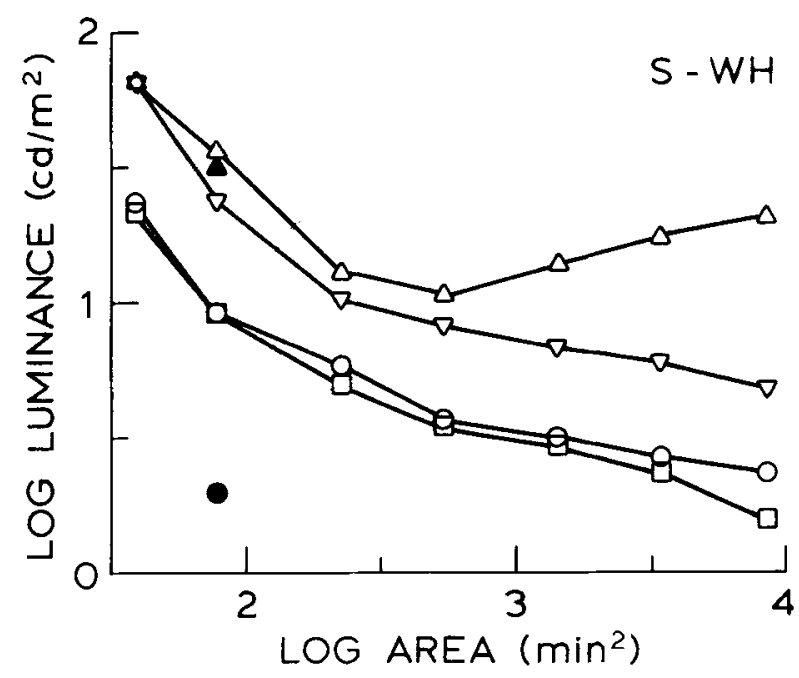

Figure 2. Averaged data from three sessions for Subject W.H. Details as in Figure 1. 


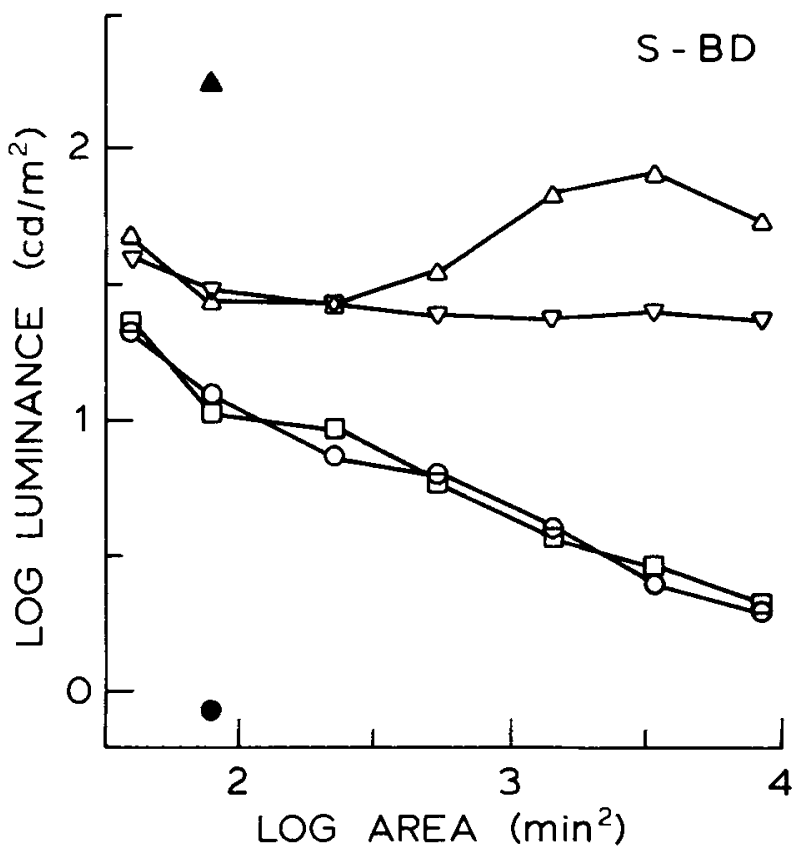

Figure 3. Averaged data from three sessions for Subject B.D. Details as in Figure 1.

\section{Variability Analysis}

Between-session variations in spatial summation functions may include both shape changes and overall sensitivity shifts. The sensitivity shifts can be effectively "factored out" by equating the function means (i.e., averages over stimulus size) for all individual sessions. Any variability that remains can then be attributed to changes of shape. This type of analysis is especially appropriate for the present data because of the hypothesis that some of the functions in Figures 1-3 are mediated by the weak channel alone and others (such as the brightness matching functions in Figures 2 and 3) are mediated by both the weak and strong channels. Single-channel functions should be relatively shape-invariant, and should thus show less residual variability than two-channel functions when overall sensitivity shifts are eliminated.

Table 1 shows an analysis of standard errors of the mean (SEs) for the data in Figures 1-3. The top row of numbers for each subject contains the average total SE (in log units) for each of the four functions, and the bottom row contains the average residual SE after normalizing the individual-session functions to their mean. The results are in good agreement with the two-channel hypothesis. Subject F.A. has roughly the same variability for all four stimulus conditions whether sensitivity shifts are included or not. In contrast, Subject B.D. shows similar total variabilities for all four conditions, but his residual (i.e., shape) variabilities are much larger for his brightness matching functions than for his threshold functions. Subject W.H. is again intermediate; his steady brightness function is more variable in shape than his threshold functions, but his flickering brightness function is not. These are the expected results if all of the threshold functions, W.H.'s flickering brightness function, and both of F.A.'s brightness functions were mediated mainly by the weak channel, but W.H.'s steady brightness function and both of B.D.'s brightness functions were mediated by both the weak and strong channels. Because the spatial summation profiles and intrinsic brightness contributions of the two channels are so different, small variations in their relative sensitivities translate into large changes in the shape of the overall brightness matching function when both channels are active.

A second effect that is apparent in Table 1 is that the residual SE is smaller for flickering than for steady brightness matches. The effect is present for all subjects, and is largest for B.D., intermediate for W.H., and smallest for F.A. Following the same type of argument as before, this suggests that flicker selectively increases the weakchannel brightness component, and thus tends to stabilize the shape of the brightness matching function. This is again the expected result if the weak channel resides in phasic neural pathways. The fact that F.A. shows some decrease may thus indicate that the strong channel provides a slight input even to his steady brightness matching function.

\section{Flicker Visibility}

All three subjects reported that flicker was visible at or very near the detection threshold for large targets but not for small targets. However, F.A. was relatively more sensitive to small target flicker than was B.D. or W.H. F.A. could usually see flicker in at least some of the brightness matching trials for even the smallest target, whereas B.D. and W.H. never could. In fact, the smallest target for which B.D. and W.H. noticed flicker during the brightness matches was the fourth (middle) target, approximately the diameter at which their flickering and steady brightness-matching curves began to diverge (see Figures 2 and 3).

A comparison of these qualitative observations with the data in Figures 1-3 suggests a complex relationship between the visibility of flicker and the activity levels of the

Table 1

Standard Errors of the Mean (SEs), Averaged Over Stimulus Size for the Data in Figure 1

\begin{tabular}{llcccc}
\hline Subject & Statistic & $\begin{array}{c}\text { Steady } \\
\text { Increment } \\
\text { Threshold }\end{array}$ & $\begin{array}{c}\text { Flicker } \\
\text { Increment } \\
\text { Threshold }\end{array}$ & $\begin{array}{c}\text { Steady } \\
\text { Brightness } \\
\text { Match }\end{array}$ & $\begin{array}{c}\text { Flicker } \\
\text { Brightness } \\
\text { Match }\end{array}$ \\
\hline F.A. & Total SE & .0815 & .0937 & .0874 & .0802 \\
& Partial SE & .0377 & .0387 & .0491 & .0279 \\
W.H. & Total SE & .0542 & .0494 & .0688 & .0404 \\
& Partial SE & .0341 & .0326 & .0663 & .0367 \\
B.D. & Total SE & .134 & .136 & .142 & .148 \\
& Partial SE & .0297 & .0202 & .0998 & .0614 \\
\hline
\end{tabular}

Note-Before partial SEs were computed, function means from individual sessions were equated to overall function means. 
postulated brightness channels. For Subject B.D., flicker has no discernible effect on threshold, and flicker must be visible to increase brightness (i.e., to lower the brightness match). In contrast, flicker appears to lower W.H.'s brightness matches, and possibly even his thresholds, for all target sizes, even though the flicker was never seen for the three smallest sizes. This result violates the well-known Talbot-Plateau law (Talbot, 1834; Plateau, 1835), which states that the appearance of a fused light (i.e., a light modulated above the critical flicker frequency) is equal to that of a comparable steady light of the same timeaverage luminance. Flicker also lowers F.A.'s brightness matches for all sizes, but it is not certain that the flicker was consistently undetectable at the brightness matching levels for small targets. Also, as discussed above, flicker effects on F.A.'s increment thresholds appear to be restricted to the four largest targets, where flicker was usually detectable at threshold.

It seems likely that the nonlinearity of the phasic system response is responsible for the brightness and sensitivity increases induced by small fused targets. The $20-\mathrm{Hz}$ frequency used in the present experiments is well within the range that typically produces the largest nonlinear effects in electrophysiological studies (e.g., see Fukada \& Saito, 1971). More importantly, the nonlinearity behaves like rectification (Victor \& Shapley, 1979), implying both a reduction of the response modulation and an increase of the average response level. When such rectification is present near threshold, it should thus be possible for flicker to produce an elevated, but unmodulated, response. If this is the right interpretation of the present results, it suggests that individual differences in the strength of the phasic system nonlinearity are not directly related to individual differences in the relative sensitivities of the phasic and tonic systems.

A number of psychophysical studies (e.g., Bowen \& Pokorny, 1978; Kitterle, 1978; Kitterle \& Corwin, 1978) have concluded that temporal brightness (or contrast) enhancement effects ${ }^{3}$ for both flashed and flickering stimuli are restricted to a neural subsystem sensitive to low spatial frequencies and high temporal frequencies. This neural subsystem is most likely the phasic system (Burbeck \& Kelly, 1981; Kelly, 1981; King-Smith \& Kulikowski, 1975). The fact that none of the subjects saw flicker in the absence of temporal brightness enhancement therefore suggests that the $20-\mathrm{Hz}$ frequency successfully discriminated against the tonic system in the present experiment.

\section{Comparison of Individual Differences in Spatial and Temporal Brightness Enhancement}

Bowen and his co-workers (Bowen \& Markell, 1980; Bowen, Sekuler, Owsley, \& Markell, 1981) found that temporal brightness enhancement ranged from robust to nonexistent in large samples of normal observers, as determined by both brightness comparison and brightness estimation procedures. Modulation sensitivity of a uniformly flickering stimulus as a function of temporal frequency, however, showed clear evidence of temporal enhancement for all observers. Bowen et al. (1981) concluded that the suprathreshold variations were probably the result of differences of criterion rather than of the neural response waveform.

The present results suggest that physiological explanations of individual differences for temporal brightness enhancement should be reconsidered. The two-channel brightness hypothesis predicts individual differences for suprathreshold, but not threshold, measures of temporal enhancement, as Bowen et al. (1981) found. Furthermore, their threshold data support the idea that the phasic system always determines the detection threshold for large stimuli. At suprathreshold levels, individual differences in the relative sensitivities of the strong and weak brightness channels can explain why some subjects show temporal enhancement but others do not. This explanation implies that spatial and temporal brightness enhancement are, to some extent, mutually exclusive for individual subjects. Specifically, subjects with a dominant strong channel should show more spatial than temporal enhancement and subjects with a dominant weak channel should show more temporal than spatial enhancement. This prediction remains to be tested.

\section{CONCLUSIONS}

(1) This study tested the hypothesis that psychophysically identified strong and weak brightness channels are mediated by tonic and phasic neural mechanisms, on the assumptions that phasic cells are more sensitive to $20-\mathrm{Hz}$ flicker than tonic cells and that flicker tends to selectively enhance the phasic response. The hypothesis predicts that $20-\mathrm{Hz}$ flicker should selectively enhance the weak-channel brightness signal. Given that the weak channel mediates most of the overall brightness signal at all target diameters for Subject F.A., predominately at large diameters for Subject W.H., and only at large diameters for Subject B.D., this is what the data in Figures 1-3 show.

(2) The variability patterns of the three subjects suggest that the shape of the brightness spatial summation function is more variable when it is determined by both the strong and weak channels than when it is determined by the weak channel alone. Also, the brightness function shape is less variable for the flickering than for the steady condition. Flicker thus appears to selectively increase the weak-channel signal, supporting the hypothesis that the weak channel is synonymous with the phasic system.

(3) The subjective reports that F.A. was relatively more sensitive to small-target flicker than B.D. or W.H. independently support the conclusion that F.A.'s phasic system was more dominant at small diameters. Nevertheless, flicker increased the brightness of small targets as much for W.H. as it did for F.A., suggesting that flicker may affect the phasic response differently for different subjects.

(4) The present findings complement studies that have found individual differences in temporal brightness enhancement (Bowen \& Markell, 1980; Bowen et al. 1981). Pending the results of direct comparative tests, individual 
differences in both spatial and temporal brightness enhancement can be explained by the two-channel brightness hypothesis.

\section{REFERENCES}

Bowen, R. W., \& MARKell, K. A. (1980). Temporal brightness enhancement studied with a large sample of observers: Evidence for individual differences in brightness perception. Perception \& Psychophysics, 27, 465-476.

Bowen, R. W., \& POKORNY, J. (1978). Target edge sharpness and temporal brightness enhancement. Vision Research, 18, 1691-1695.

Bowen, R. W., Sekuler, R., OWsley, C. J., \& Markell, K. A. (1981). Individual differences in pulse brightness perception. Perception \& Psychophysics, 30, 587-593.

BrocA, D., \& Sulzer, A. (1902). La sensation lumineuse en fonction du temps. Journal de Physiologie et de Pathologic Generale, 4, 632-640.

BurbeCK, C. A., \& KellY, D. H. (1981). Contrast gain measurements and the transient/sustained dichotomy. Journal of the Optical Society of America, 71, 1335-1342.

Cleland, B. G., Levick, W. R., \& Sanderson, K. J. (1973). Properties of sustained and transient ganglion cells in the cat retina. Journal of Physiology (London), 228, 649-680.

DeMonasterio, F. M. (1978). Properties of concentrically organized $X$ and $Y$ ganglion cells of Macaque retina. Journal of Neumphysiology, 41, 1394-1417.

DRUM, B. (1976). The relation of apparent brightness to contrast threshold on a photopic background: Dependence on retinal position and target size. Vision Research, 16, 1401-1406.

DRUM, B. (1980). Relation of brightness to threshold for light-adapted and dark-adapted rods and cones: Effects of retinal eccentricity and target size. Perception, 9, 633-650.

FuKADA, Y., \& SarTo, H.-A. (1971). The relationship between response characteristics to flicker stimulation and receptive field organization in the cat's optic nerve fibers. Vision Research, 11, 227-240.

Gouras, P. (1968). Identification of cone mechanisms in monkey ganglion cells. Journal of Physiology, 199, 533-547.

HAMMON, R. W., \& SCOBEY, R. P. (1982). The luminance and response range of monkey retinal ganglion cells to white light. Vision Research, 22, 271-277.

Higgins, K. E., \& RiNalducCI, E. J. (1975). Suprathreshold intensityarea relationships: A spatial Broca-Sulzer effect. Vision Research, 15 129-143.

KELLY, D. H. (1981). Nonlinear visual responses to flickering sinusoidal gratings. Journal of the Optical Society of America, 71, 1051-1055.

KING-Smith, P. E., \& KuLIKowsKI, J. J. (1975). Pattern and flicker detection analysed by subthreshold summation. Journal of Physiology (London), 249, 519-548.
KITTERLE, F. L. (1978). Contrast enhancement: Influence of spatial frequency and flicker rate. Journal of the Optical Society of America, 68, 1438-1439.

KitTerle, F. L., \& CoRWIN, T. R. (1978). Enhancement of apparent contrast in flashed sinusoidal gratings. Vision Research, 19, 33-39.

Lennie, P. (1980). Parallel visual pathways: A review. Vision Research, 20, 561-594.

Plateau, J. (1835). Sur un principe de photométrie. Academie Royal des Sciences, Brussels, Bulletin, 2, 52-59.

SLOAN, L. L. (1971). The Tübinger perimeter of Harms and Aulhorn. Archives of Ophthalmology, 86, 612-622.

TALвot, H. F. (1834). Experiments on light. Philosophical Magazine, 5, 321-334.

VICTOR, J. D., \& SHAPLEY, R. M. (1979). The nonlinear pathway of Y ganglion cells in the cat retina. Journal of General Physiology, 74, 671-689.

\section{NOTES}

1. Different investigators have used different criteria to distinguish two classes of retinal ganglion cells or lateral geniculate nucleus cells, which are variously known as $\mathrm{X}$ and $\mathrm{Y}$, tonic and phasic, or sustained and transient. Although these classifications do not agree perfectly, they are consistent enough to indicate two common underlying types of neurons with complementary spatial and temporal properties. I have followed the tonicphasic terminology in this paper, after Gouras (1968), who first described the two classes in the primate.

2. Subjects sometimes failed to see the dimmest test stimuli in the brightness matching experiment. Previous studies (Drum, 1976, 1980) had shown that peripheral threshold stimuli can appear brighter than a suprathreshold foveal stimulus; automatically labeling unseen targets as "dimmer" could thus tend to overestimate the matching luminance. To avoid this bias, trials for which the subject did not see the stimulus were ignored and randomly repeated later in the sequence.

3. The term "enhancement" is widely used to describe a number of nonmonotonic relationships between brightness or contrast and various spatial or temporal variables. Most of the phenomena to which they refer (including the Broca-Sulzer effect and its spatial analog) are actually thought to arise from the temporary or localized loss of antagonistic interactions rather than from a true enhancement mechanism. Flickerinduced brightness increases, however, may be true enhancement effects to the extent that they arise from the nonlinearity of the phasic system response (see text).

(Manuscript received August 15, 1983; revision accepted for publication July 6,1984 .) 